\title{
Image representation method based on Complex Wavelet Transform and Phase Congruency, with Automatic Threshold Selection
}

\author{
Arathi T \\ PhD Scholar, Department of Computer Science \& \\ Engineering \\ Amrita Vishwa Vidyapeetham \\ Coimbatore, India \\ arathiviswam09@yahoo.co.in
}

\author{
Latha Parameswaran \\ Professor, Department of Computer Science \& Engineering \\ Amrita Vishwa Vidyapeetham \\ Coimbatore, India \\ p_latha@cb.amrita.edu
}

Received: June 28, 2019. Revised: August 3, 2021. Accepted: September 11, 2021. Published: November 27, 2021.

\begin{abstract}
Image representation is an active area of research with increasing applications in military and defense. Image representation aims at representing an image with lesser number of coefficients than the actual image, without affecting the image quality. It is the first step in image compression. Once the image is represented by using some set of coefficients, it is further encoded using various compression algorithms. This paper proposes an adaptive method for image representation, which uses Complex Wavelet transform and the concept of phase congruency, where the number of coefficients used for image representation depends on the information content in the input image. The efficiency of the proposed method has been assessed by comparing the number of coefficients used to represent the image using the proposed method with that used when Complex Wavelet transform is used for image representation. The resultant image quality is determined by computing the PSNR values and Normalized Cross Correlation. Experiments carried out show highly promising results, in terms of the reduction in the number of coefficients used for image representation and the quality of the resultant image.
\end{abstract}

Keywords- Image Representation; Complex Wavelet Transform; Coefficient of Variation; Phase Congruency; Peak Signal to Noise Ratio

\section{INTRODUCTION}

Transforming images from the spatial domain to the frequency domain has been found to be the general trend followed in various representation methods. The key reason for this is the way frequency domain representation of the image makes the image coefficients uncorrelated with each other, making their analysis easier. The commonly used tool for converting the image data from spatial to spectral domain is the Fourier transform [1]. Due to the non-local property of Fourier transform, they were replaced by wavelet functions, which due to their unique property of being local [2], was found to be better suited for data representation. Wavelets were found to be highly efficient in approximating data with sharp discontinuities [2]. The advent of wavelets opened a new path for the development of image representation algorithms. The availability of a 1 arge variety of wavelets allowed the image to be analyzed at multiple resolutions, thereby allowing the redundancy to be removed from each resolution level. However, even though Discrete Wavelet Transform (DWT) proved promising, it has inherent limitations. Since the DWTs are critically sampled, it is not shift invariant and lacks directional selectivity. This led to the development of a variant of the conventional wavelet called the Complex Wavelet Transform (CWT) [3]. This makes the transform shift invariant, but the directional selectivity is still a problem in CWT.

Phase Congruency is a feature operator which is invariant to illumination and scale. It assumes an image to be highly rich in information and very little redundancy. This property makes sure that the proposed technique doesn't treat any major information in the image as redundant and remove it [4].

This paper combines CWT and the concept of Phase Congruency and proposes a new technique for image representation. Even though image representation techniques aim at reducing the number of coefficients used for representing the image [5], it also results in loss of information in the image. Hence, a trade-off must be obtained between the reduction in the number of coefficients obtained and the quality of the resultant image. A good representation algorithm should aim to obtain a fairly good trade-off between the two. The percentage of reduction in the coefficients and the resultant image quality $[6,7]$ are also dependent on the type of the input image. The proposed technique adaptively changes the threshold value for redundancy removal, depending on the input image. Experimental results show that the proposed method achieves very good levels of coefficient reduction and at the same time does not compromise much on image quality, as the information loss is kept to the bare minimum.

\section{COMPLEX WAVELET TRANSFORM}

The Discrete Wavelet Transform has the following drawbacks [3]:

- DWT coefficients oscillate at zero crossings;

- It is not shift invariant; 
- $\quad$ Aliasing occurs due to downsampling during analysis, which is not always cancelled during synthesis;

- DWT lacks directional selectivity;

CWT employs quadrature wavelets, which provides a magnitude and phase representation, shift invariance and no aliasing as well.

CWT can be expressed as:

$$
\psi_{c}(t)=\psi_{r}(t)+j \psi_{i}(t)
$$

where, $\psi_{r}(t)$ is real and even and $j \psi_{i}(t)$ is imaginary and odd. If $\psi_{r}(t)$ and $\psi_{i}(t)$ are chosen to be out of phase with each other by $90^{\circ}$ (Hibert transform pair), then $\psi_{c}(t)$ is an analytic signal and supported on only one-half of the frequency axis. The complex wavelet coefficients can be expressed as:

with magnitude

$$
d_{c}(j, n)=d_{r}(j, n)+j d_{i}(j, n)
$$

$$
\left|d_{c}(j, n)=\sqrt{\left|d_{r}(j, n)\right|^{2}+\left|d_{i}(j, n)\right|^{2}}\right|
$$

and phase angle given by

$$
\angle d_{c}(j, n)=\arctan \left(\frac{d_{i}(j, n)}{d_{r}(j, n)}\right)
$$

CWT enables to analyse and represent both real-valued and complex-valued signals, just like Fourier transform [18].

\section{A. DT-CWT Filterbank}

The filterbank structure of the 1-D, DT-CWT resembles the conventional DWT, with twice the complexity [8]. It can be thought of as two conventional DWT trees operating in parallel. One is the real tree and the other is the imaginary tree. The conjugate filters used in analysis are of the form: $h_{x}+j g_{x}$, where $h_{x}$ is the set of filters $\left\{h_{0}, h_{1}\right\}$ and $g_{x}$ is the set of filters $\left\{g_{0}, g_{1}\right\}$. Figure 1 shows that the filters $h_{0}$ and $h_{1}$ are the real-valued lowpass and highpass filters respectively for the real tree. $g_{0}$ and $g_{1}$ are the real-valued lowpass and highpass filters respectively for the imaginary tree.

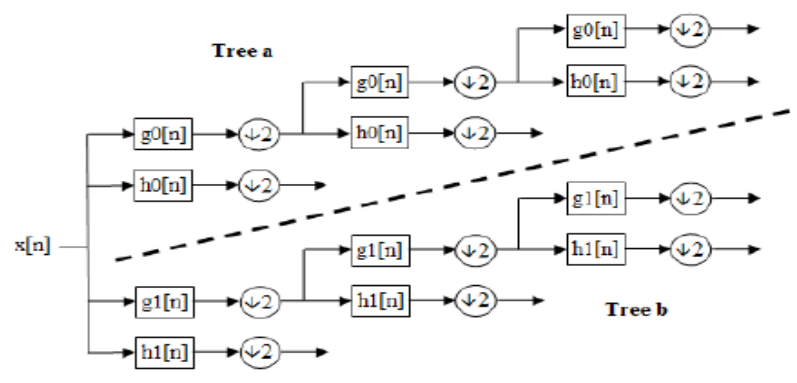

Figure 1: DT-CWT structure with two separable DWT
The synthesis filter pairs form orthogonal pairs with their respective counterparts of the analysis tree. For the 2D DT-CWT, the filter structure has four trees for analysis and synthesis as shown in Figure 2. The pair of conjugate filters is applied to two dimensions ( $\mathrm{x}$ and $\mathrm{y}$ ) and is expressed as:

$$
\left(h_{x}+j g_{x}\right)\left(h_{y}+j g_{y}\right)=\left(h_{x} h_{y}-g_{x} g_{y}\right)+j\left(h_{x} g_{y}+g_{x} h_{y}\right)
$$

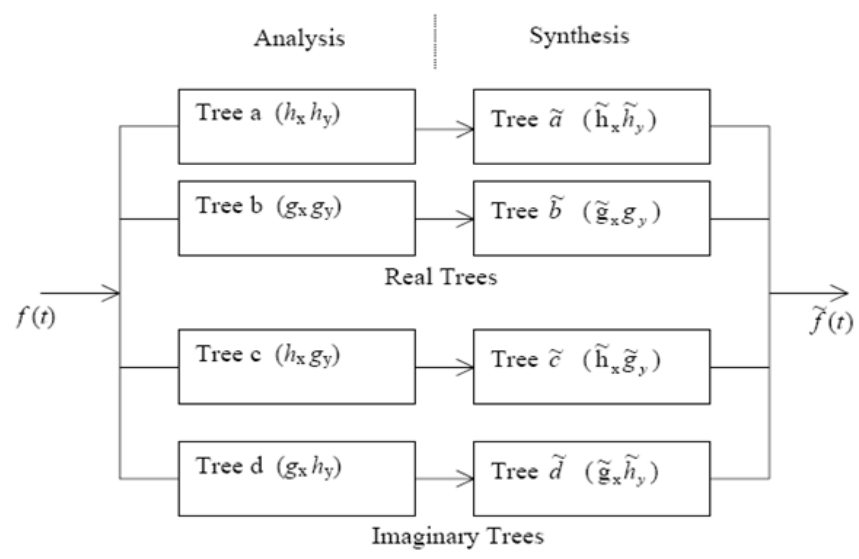

Figure 2: Filter bank structure for 2-D DT-CWT

The 2-D DT-CWT is 4-times expensive than the standard 2-D DWT, since it has 4 different trees. Trees $\boldsymbol{a}$ and $\boldsymbol{b}$ are the real pair and trees $\boldsymbol{c}$ and $\boldsymbol{d}$ form the imaginary pair in the analysis stage. Tree pairs $(\tilde{a}, \tilde{b})$ and $(\tilde{c}, \tilde{d})$ are the real and imaginary parts respectively in the synthesis stage, corresponding to the analysis pairs [9].

\section{Phase CONGRUENCY}

It has been traditionally a practice in image processing to think about features in terms of derivatives. This is because, features in images are mostly thought of as edges, which are points of discontinuities. As a result, gradient based operators are mostly used to detect the features in images. The gradient based feature estimation techniques, such as those developed by Sobel [11], Marr and Hildreth [12] and Canny [13, 14], face two major drawbacks. Firstly, the gradient operators are sensitive to illumination variations. i.e; they cannot be relied on, when working with images of varying lighting and contrast. The second shortcoming of gradient operators is that, localization of features depends on the scale of analysis. Hence, the localization becomes innacurate when analysed at varying scales. This leads to the need of a feature operator that is invariant to illumination and scale.

Phase congruency model of feature detection [4] assumes an image to be high in information and low in redundancy. Thus, instead of searching for points of sharp changes in intensity, this model searches for patterns, where the phase components of the Fourier transform of the image are in order (maximally in-phase). It is a f requency-based model and instead of spatial processing of data, it processes an image using the phase and amplitude components of the individual frequency components. 
Consider a 1-D slice through an image. Such a signal $f(x)$ can be reconstructed from its Fourier transform by:

$$
f(x)=\int_{-\infty}^{\infty} a_{\omega} \cos \left(T \omega x+\phi_{\omega}\right) d \omega,
$$

where for each frequency $\omega, a_{\omega}$ is the amplitude of the cosine wave and $T \omega x+\phi_{\omega}$ is the phase offset of that wave. The term ' $\boldsymbol{T}$ ' is related to the size of the image window. The phase congruency model in the discrete form is expressed as:

$$
f(x)=\sum_{n=0}^{\infty} \frac{1}{(2 n+1)^{p}}[\sin (2 n+1) x+\phi],
$$

where, $\boldsymbol{p}$ gives the decay in the amplitude with frequency and $\phi$ is the phase offset. Phase Congruency is the ratio of local energy to amplitude. $P C(x)=\frac{|E(x)|}{\sum_{n} A_{n}(x)}$, where, $A_{n}(x)$ is the amplitude and $E(x)$ is the local energy and $0 \leq P C(x) \leq 1[10]$.

\section{PROPOSED TECHNIQUE FOR IMAGE REPRESENTATION}

In $[9,10]$ the authors have discussed an image representation technique using CWT and Wavelet transform. This proposed technique de-correlates the input image information by transforming the image into the frequency domain using CWT. The proposed algorithm makes use of the concept of phase congruency, to determine the amount of redundant information that needs to be removed from the input image. In our earlier paper [15], we had proposed a technique for image compression, which uses Slantlet transform and phase congruency, where the threshold had to be provided by the user, which determined how much of the coefficients would be removed for a fairly good representation. However, choosing threshold values empirically can't always be reliable, as the amount of redundancy is image dependent.

We have introduced a threshold selection method in this paper, which selects the threshold value automatically from the input image, based on its information content. Here the threshold value used is the Coefficient of Variation [16, 17] of the transformed image. The value thus obtained is found to be an optimum threshold, which reduces the number of coefficients used for representation by almost $60-70 \%$, at the same time maintaining the image quality.

The algorithm is applied on the image as a whole. The following steps are carried out on the input image.

\section{A. Decomposition of theiInput image using CWT}

Complex Wavelet transform is applied to each column of the input image. The CWT filter coefficients used in this experimentation is obtained from [3]. Let $I(x, y)$ be the input block to be compressed. The CWT of each column of the block is carried out, resulting in a corresponding coefficient block in the transform domain, denoted as $T_{I}(u, v)$. Let the transform domain coefficients of the transformed block be represented as $C(u, v)_{T I}$.

\section{B. Phase Congruency map for the decomposed Image}

The next step in the proposed compression technique is to create the phase congruency map for the transformed image block, $T_{I}(u, v)$. Each transform domain coefficient will thus have a phase congruency value corresponding to the position of the coefficient in the transformed image. Let the phase congruency map for the transformed image block be denoted as $P C_{T I}$ and each phase congruency value be represented as $P C(u, v)_{T I}$.

\section{Selecting the threshold value for selection of coefficients Figures and Tables}

Once the phase congruency map has been generated, we need to select the threshold value for selecting the coefficients. We select the threshold value by computing the Coefficient of Variation [16] for the transformed image. The value for the threshold is obtained as:

$$
T_{S H}=\frac{\sum_{i=1}^{m n} C(u, v)_{T I}}{m n}
$$

\section{Removing the redundant CWT coefficients Figures and Tables}

The phase congruency map acts as the basis for removing the redundant CWT coefficients. The compression algorithm chooses only those CWT coefficients from the transformed image block, which has edge strength greater than the obtained threshold $T_{S H}$. The edge strength is represented by the normalized phase congruency value from the phase congruency map. The decision rule can be expressed as:

$$
\begin{aligned}
& C^{C}(u, v)_{T I}=C(u, v)_{T I} \text {, if } \quad P C(u, v)_{T I}>T_{S H} \\
& =0, \quad \text { if } \quad P C(u, v)_{T I}<T_{S H}
\end{aligned}
$$

where, $C^{C}(u, v)_{T I}$ are the CWT coefficients of the compressed image.

\section{E. Obtaining the final mage block}

To get the resultant image block, the inverse Complex Wavelet transform of the coefficients thus selected is taken. The final result is expressed as:

$$
F_{\text {IC }}(x, y)=\operatorname{IST}\left(C^{C}(u, v),\right.
$$

where, $F_{I C}(x, y)$ is the final image block. It can be observed that the number of CWT coefficients that were used to reconstruct back the final image block is much lesser than the actual number of CWT coefficients that was used to represent the input image block.

Experimental results also prove that this technique helps to achieve almost $60-70 \%$ reduction in the number of coefficients used for representing the image, without trade-off in the visual quality of the image. 


\section{F. Experimental Results and Analysis}

The proposed CWT and phase congruency based image representation technique was applied on a s et grayscale images of size 512 x 512 of various categories such as standard test images, natural images, user created images and medical images. The number of coefficients used for representation, PSNR and Normalized Cross Correlation (NCC) values are computed to determine the degree of efficiency achieved in representing the image and the amount of information loss respectively. Experimental results pertaining to six images of various categories are discussed here. Figure 3 shows the input images used and Figure 4 shows the output images of the proposed method. It can be observed that the visual quality of the image has not degraded due to this technique.
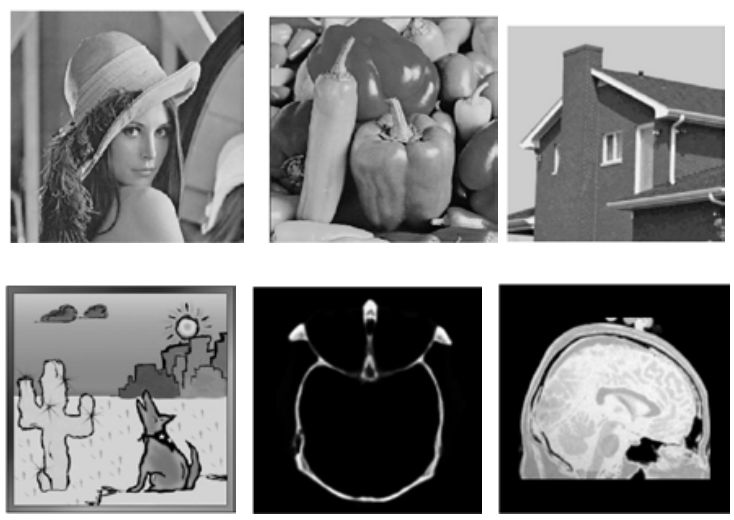

Figure 3: Input Images
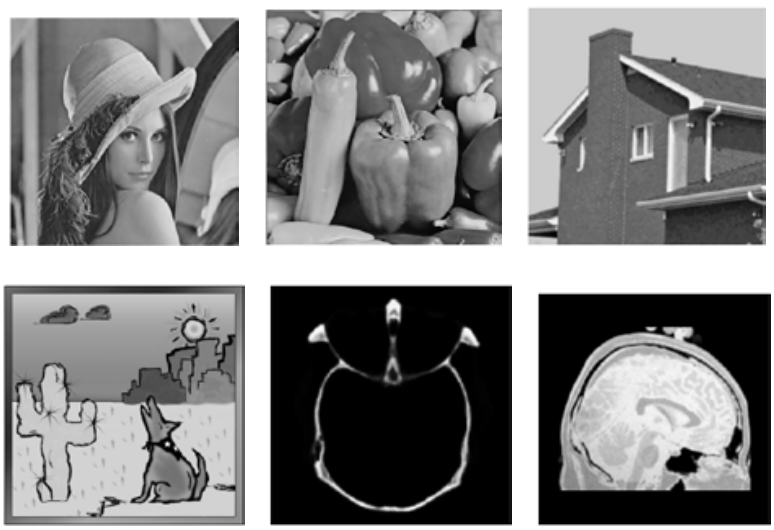

Figure 4: Resultant images using the proposed method

For analyzing the performance of the proposed method, percentage reduction in the number of coefficients when compared to conventional CWT, PSNR and NCC values are measured. In each case the number of CWT coefficients required to represent the image and the number of coefficients required to represent the same image, after the proposed method have been used for analysis. Table 1 shows the details regarding the number of coefficients in CWT based image representation, the resultant coefficients after the proposed technique, percentage reduction in the number of coefficients used for representation in the proposed method, PSNR and NCC. It also shows the automatically generated threshold for each input image. It also shows the automatically generated threshold for each input image.

TABLE I. NO: OF COEFFICIENTS USED FOR REPRESENTATION IN CONVENTIONAL CWT AND THE PROPOSED METHOD, ITS PERCENTAGE EQUIVALENT, PSNR AND NCC VALUES.

\begin{tabular}{|c|c|c|c|c|c|c|}
\hline Observations & Lena & Peppers & Natural & $\begin{array}{c}\text { User } \\
\text { Created }\end{array}$ & CT & MRI \\
\hline CWT & 301401 & 301401 & 301401 & 301401 & 301401 & 301401 \\
\hline $\begin{array}{c}\text { Proposed } \\
\text { Method }\end{array}$ & 86482 & 79086 & 84029 & 109956 & 116339 & 125178 \\
\hline $\begin{array}{c}\text { No: of } \\
\text { coefficients } \\
\text { in \% }\end{array}$ & $28.69 \%$ & $26.24 \%$ & $27.88 \%$ & $36.48 \%$ & $38.60 \%$ & $41.53 \%$ \\
\hline $\begin{array}{c}\text { Automatic } \\
\text { Threshold } \\
\text { Value }\end{array}$ & 0.1611 & 0.1590 & 0.1818 & 0.1912 & 0.0117 & 0.1053 \\
\hline PSNR & 37.2480 & 34.7208 & 45.5925 & 35.9257 & 45.9276 & 41.4324 \\
\hline NCC & 0.9954 & 0.9947 & 0.9961 & 0.9953 & 0.9967 & 0.9960 \\
\hline
\end{tabular}

From Table 1 it is clear that, using the proposed method, the input image can be represented with almost only $30-40 \%$ of coefficients, as with conventional CWT. There is no much compromise on the image quality. Visual comparison of the original and the resultant image shows that there is no visual change between the two, ascertaining excellent visual quality. The values of PSNR are high, which shows that there is no much loss of information in the proposed method. Also, the NCC value is very close to 1 , which tells that the resultant image obtained is $99 \%$ same as the original image.

\section{CONCLUSION}

Complex wavelet transform, removes the shortcomings of the conventional DWT. Hence, it has the advantages of shift invariance, directionality and it also avoids aliasing to a large extent. CWT can thus be thought of as a powerful tool in multiresolution analysis. The phase congruency map is generated from the CWT coefficients of the input image and is used as the decision rule to find out the coefficients that are used for image representation. The threshold to be used for the selection of coefficients is obtained automatically from the input image. Coefficients of variation is used for selection of the threshold, as it considers the information content in the image and selects an appropriate threshold value, which doesn't remove any relevant information in the image. Exhaustive experiments conducted on grayscale images exhibit promising results. The experimental analysis of the results thus obtained shows that using the proposed method, a high degree of reduction in the number of coefficient for representing an image can be obtained, when compared to conventional Complex Wavelet transform. 


\section{REFERENCES}

[1] Eric W. Weisstein, "Fourier Transforms" From Mathworld-a Wolfram Web Resource. http://mathworld.wolfram.com/FourierTransforms.html.

[2] M. Sifuzzaman1, M.R. Islam1 and M.Z. Ali, "Application of Wavelet Transform and its Advantages Compared to Fourier Transform", Journal of Physica Sciences, Vol. 13, Pages 121-134, 2009.

[3] I. W. Selesnick, R. G. Baraniuk, and N. G. Kingsbury, "The dualtree complex wavelet transform," IEEE Signal Processing Magazine, vol. 22, no. 6, pp. 123-151, November 2005.

[4] P. D. Kovesi. A dimensionless measure of edge significance from phase congruency pages calculated via wavelets. International First New Zealand Conference on Image and Vision Computing, Auckland, August 1993.

[5] Wei Hong, John Wright, Kun Huang, Yi Ma, Multi-Scale Hybrid Linear Models for Lossy Image Representation. IEEE Transactions on Image Processing, 2006.

[6] Deepak S. Turaga, Yingwei Chen, Jorge Caviedes, "No reference PSNR estimation for compressed pictures, Signal Processing: Image Communication, Elsevier, Vol. 19, Pages: 173-184, 2004.

[7] Tania Stathaki, "Image Fusion: Algorithms and Applications", Academic Press, 2008 edition.

[8] Felix Fernandes, 'Directional, Shift-insensitive, Complex Wavelet Transforms with Controllable Redundancy', PhD Thesis, Rice University, 2002.

[9] Dr. Salih Husain Ali \& Aymen Dawood Salman, Image Compression Based on 2D Dual Tree Complex Wavelet Transform (2D DT-CWT), Enggineering \& Technical Journal, Vol. 28, No.7, 2010.
[10] M.B. Pardo, C.T. van der Reijden, "E mbedded lossy image compression based on wavelet transform", Video/Image Processing and Multimedia Communications 4th EURASIP- IEEE Region 8 International Symposium on VIPromCom, November 2002.

[11] J. F. Canny. A computational approach to edge detection. IEEE Transactions on Pattern Analysis and Machine Intelligence, 8(6):112$131,1986$.

[12] R. Deriche. Using Canny's criteria to derive an optimal edge detector recursively implemented. The International Journal of Computer Vision, 1:167-187, April 1987.

[13] D. L. Donoho. De-noising by soft thresholding. Technical Report 409, Department of Statistics. Stanford University, 1992.

[14] D. J. Field. Relations between the statistics of natural images and the response properties of cortical cells. Journal of the Optical Society of America A, 4(12):2379-2394, December 1987.

[15] Arathi T, Latha Parameswaran, Slantlet Transform and Phase congruency based Image Compression, AICWIC'13, Proceedings published by International Journal of Computer Applications, IJCA, January 2013.

[16] Anthony Tanbakuchi, Introductory Statistics Lectures-Measures of Variation, 2009.

[17] S.E Ahmed, A Pooling Methodology for Coefficient of Variation, The Indian Journal of Statistics, Volume 57, Series B, pages 57-75, 1995.

[18] N G Kingsbury: "Complex wavelets for shift invariant analysis and filtering of signals", Journal of Applied and Computational Harmonic Analysis, vol 10, no 3, May 2001, pp. 234-253.

\section{Creative Commons Attribution License 4.0 (Attribution 4.0 International, CC BY 4.0)}

This article is published under the terms of the Creative Commons Attribution License 4.0

https://creativecommons.org/licenses/by/4.0/deed.en_US 